\title{
A Study in Notched Beam Stress Concentration
}

\author{
Raphaël Cordina, Mechanical Engineering, Aderet Pantierer, Mechanical Engineering, Shmuel Pantierer, Mechanical \\ Engineering, Nicolas Ceballos, Mechanical Engineering \\ Vaughn College of Aeronautics and Technology, NY, USA, raphael.cordina@ vaughn.edu, aderet.pantierer@vaughn.edu, \\ shmuel.pantierer@vaughn.edu,nicolas.fdo.cg@gmail.com
}

Mentor: Yougashwar Budhoo, Ph.D., Hossein Rahemi, Ph.D.

Vaughn College of Aeronautics and Technology, NY, USA, yougashwar.budhoo@vaughn.edu, hossein.rahemi@vaughn.edu

\begin{abstract}
Mechanical analysis of a load bearing structure or component can be tedious or straightforward depending on the loading conditions and geometry. One-dimensional structure analytical techniques are usually the go-to approach due to their simplicity. However, if there is a discontinuity in the component, there is an amplification in the stress and a stress concentration factor is applied to find the true maximum, or critical, stress experienced. This factor is found on published graphs produced from historical data. Stress concentration graphs exist for axial loads or bending loads, but not for combined loading. This paper seeks to investigate the effect of combined loading on stress concentration and create a stress concentration graph for a notched object undergoing bending and axial loading simultaneously. A series of analyses are performed using a finite element analysis software to obtain the values required to create the graph. The study consists of analyzing four notched beams, each undergoing five iterations of testing. Each beam is designed with the same overall dimensions, excluding in notch radius size. The resulting values are then used to create a stress concentration graph for a notched beam undergoing combined loading.
\end{abstract}

Keywords - Stress Concentration, Combined Loading, Finite Element Analysis.

\section{INTRODUCTION}

Engineers are tasked with creating machines, and subsequently parts, that are subjected to numerous conditions due to several variables, including harsh weather and varying loading requirements. While the average person may be able to design a simple part to withstand a load, it will likely be overengineered and cumbersome. Using various mathematical formulae and methods, engineers can develop a streamlined part that uses fewer resources and has a lower production cost, all the while enabling the component to withstand the loading it will face.

One of these formulae is the equation for stress, defined as "the force per unit area, or intensity of the forces distributed over a given section" [4]. Knowing this value allows the engineer to adjust the part's dimensions to better suit the calculated stress the component can withstand. However, if the part in question has a discontinuity, such as a hole, notch, or crack, the experienced stress is magnified and concentrated at the discontinuous area. This magnification is known as stress concentration.

In order to determine the amplified stress, the calculated stress must be manipulated to produce this value. A stress concentration factor, $K$, is used to relate the calculated stress and the maximum stress, found at the discontinuity [5]. This Digital Object Identifier (DOI):

http://dx.doi.org/10.18687/LACCEI2019.1.1.492

ISBN: 978-0-9993443-6-1 ISSN: 2414-6390

$1^{\text {th }}$ LACCEI International Multi-Conference for Engineering, Education, and Technology: "Industry, Innovation, And Infrastructure for Sustainable Cities and Communities”, 24-26 July 2019, Jamaica. dimensionless factor determines the magnitude of stress a part experiences at a discontinuity.

The stress concentration factor can be found in multiple ways, both analytically and experimentally. In order to find $K$ analytically, both the actual stress and maximum stress must already be known. If an experiment were to be performed on the part in question, strain gages could be attached at the points of discontinuity which would in turn yield the results of the maximum stress produced. However, most lack the resources to perform such experiments and therefore utilized programs that conduct Finite Element Analysis, FEA [6].

Another method of finding $K$ is through the use of stress concentration graphs. These graphs were produced for a limited amount of geometries and loading conditions. From the graphs, $K$ is determined with only the knowledge of the geometrical dimensions of the part to be analyzed. The creation of these graphs required the knowledge of both the actual stress and the maximum stress, something that is only found through experimentation or the use of a stress concentration factor. Therefore, various tests were performed to determine the values to be plotted. These tests analyzed the stress a component experiences under various loading conditions [5]. However, the analyses, and thereby the graphs, only consider axial or bending loading conditions, never combined loading conditions. A graph representing the stress concentration factor under combined loading is not currently available. While this limitation may be acceptable in the academic world, values for combined loading are necessary for a real-world application.

\section{OBJECTIVE}

This experiment is performed to create a stress concentration graph that will produce valid stress concentration factor values from combined loading conditions. A notched beam is analyzed using the Finite Element Method, FEM, while undergoing axial and bending loading to find the value of stress experienced by the part. Lacking the expensive equipment and hardware necessary to run the tests physically, CATIA is used to model and analyze the component.

Two independent variables are currently used to determine the characteristics of the curves of stress concentration graph already produced. These parameters are the ratio between the width of the beam, $W$, to the distance between the notches, $d$, and the relationship between the radius of the notch, $r$, to the distance between the two notches. These ratios are 
further referenced as $W / d$ and $r / d$, respectively. The variables of the dimensions of the beam can be seen in Fig. 1. The values of the ratios used in this experiment are developed by referencing stress concentration graphs for individual loading [5]. This was done to allow the ratios to remain consistent throughout each experiment and trial. These ratios also dictate the geometry of the beam. With the values of the ratios defined, the dimensions of the beam are calculated.

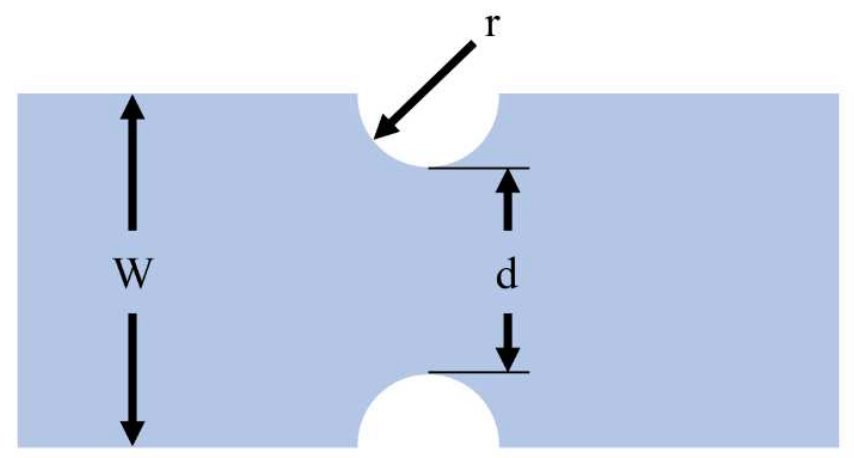

Fig. 1 Dimensions used in the ratios dictating the values of the stress concentration graph

Four different beams are analyzed to produce a stress concentration graph with four unique curves. The four beams were each designed to have a uniform width. The distance between the notches varied between the four different tests but remain uniform for each individual beam. The variable value that produces the plot points of the curves is the radius of the notches for each of the five trials; this value is changed for each test. From the results obtained through the analyses, the values of the stress concentration factor are found, and the graph is created.

The reason for creating this graph is so that, in the future, tests do not have to be performed to determine the $K$ value required for combined loading. Instead, the only values necessary are the radius of the notch, the width of the beam, and the distance between the notches. The availability of this graph would enable a more conducive workflow because numerous experiments would not need to be conducted to find the appropriate stress concentration factor.

\section{ANALYSIS}

A.

\section{Geometry}

The beams are modeled using the CATIA software. Each experiment uses the same width of $7.62 \mathrm{~cm}(3$ in), thickness of $1.27 \mathrm{~cm}(0.5 \mathrm{in})$, and length of $30.48 \mathrm{~cm}$ (12 in). The beams are constrained on one face to simulate the reactionary forces a wall would produce. A tensile load of $1334.47 \mathrm{~N} \mathrm{(300}$ lbs) and a moment about the transverse axis of $16.95 \mathrm{~N}-\mathrm{m}(150$ in-lb) are applied to the free end of each beam. This loading remains consistent throughout all the experiments and trials so that the maximum stress can be computed using the identical loading conditions. These governing parameters can be seen in Fig. 2.

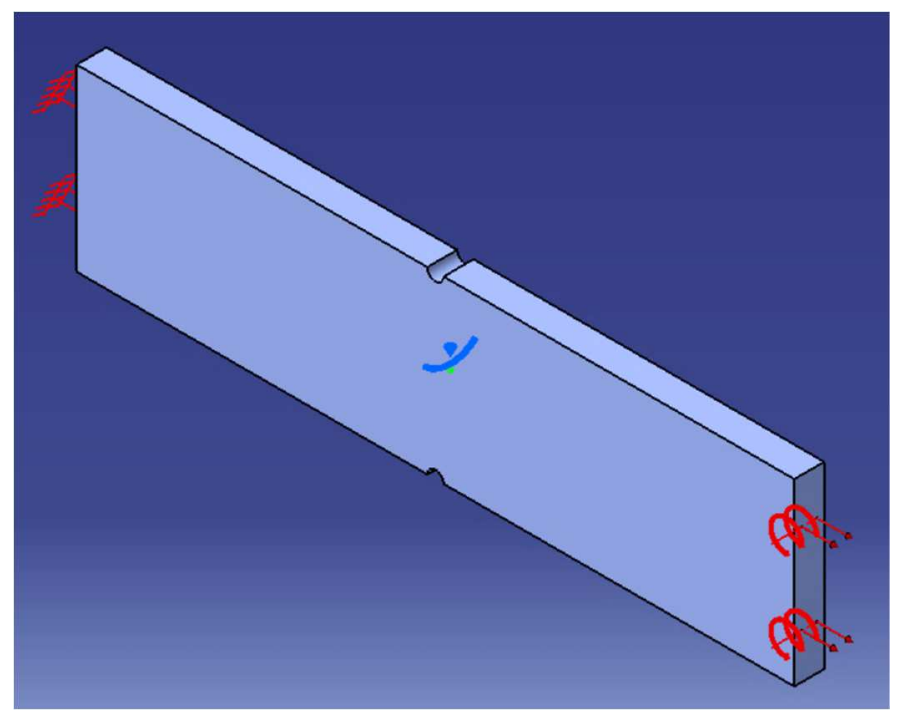

Fig. 2 Geometry of the beam with loading conditions applied to the right face and a fixed constraint on the left face. [3]

Four experiments are performed, each governed by their respective $W / d$ ratios. These ratios must remain consistent so the stress concentration curves can be dictated by uniform variables. The $W / d$ ratios are selected to be $3,1.5,1.2$, and 1.1 in reference to the separate stress concentration graphs for axial and bending loads [5]. Every beam undergoes five iterations of tests with the same independent variable for each experiment: the ratio between the radii of the notches to the distance between them, $r / d$. The ratio is chosen to begin at 0.05 and increase to 0.25 in increments of 0.05 . These values are determined with respect to existing stress concentration graphs, as can be seen in Fig 3. and Fig 4. Each experiment uses the same $r / d$ ratios, but due to the $W / d$ ratio differing per experiment, the individual radii change accordingly from trial to trial within each of the four tests.

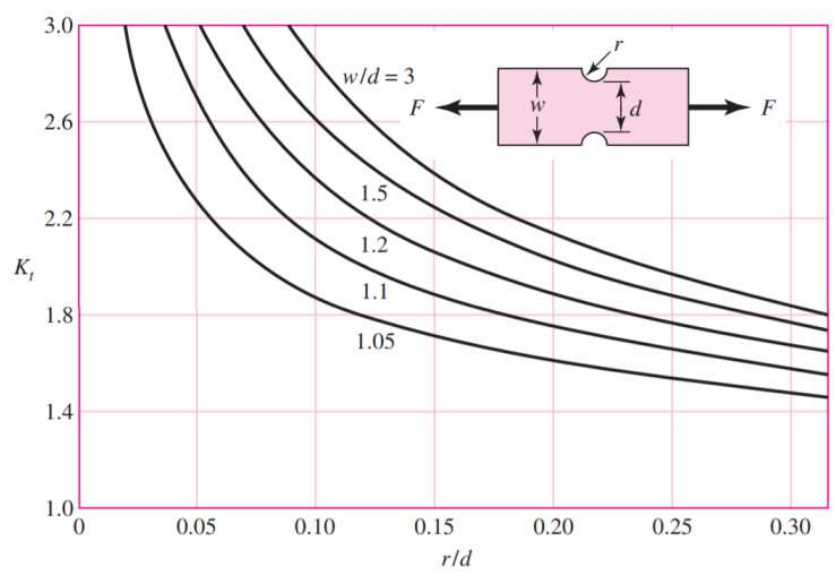

Fig. 3 Existing stress concentration chart for a notched beam experiencing tensile loading. [2]

$17^{\text {th }}$ LACCEI International Multi-Conference for Engineering, Education, and Technology: "Industry, Innovation, And Infrastructure for Sustainable Cities and Communities", 24-26 July 2019, Jamaica. 


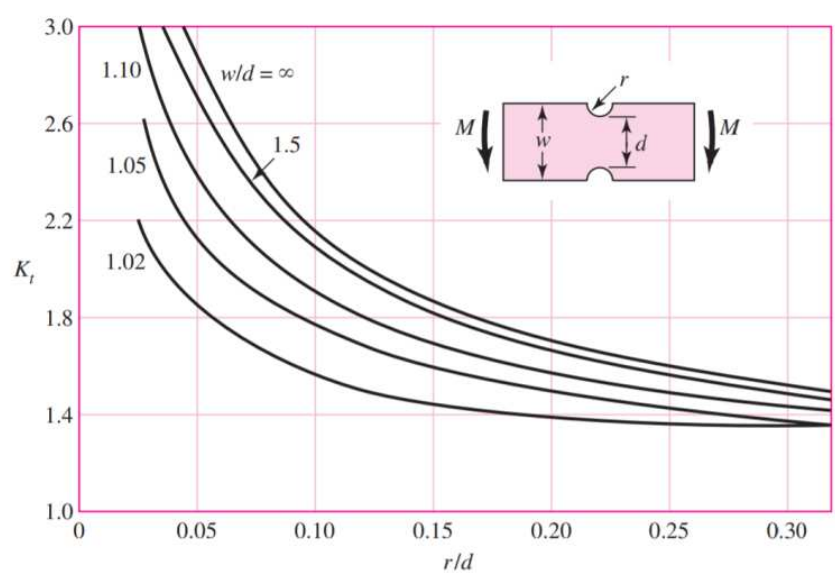

Fig. 4 Existing stress concentration chart for a notched beam experiencing transverse bending. [2]

B. $\quad$ Testing of Beams with W/d Ratios of 3 and 1.5

For the first two experiments, using the $W / d$ ratios of 3 and 1.5 , the aforementioned calculated values of radii are used and produce reliable results. The entire radius of the notch is within the geometry of the beam, with tangential lines protruding to the edges of the beam, see Fig. 5. As the notch radius increases, the notch edges decrease and move closer to the horizontal sides of the beam.

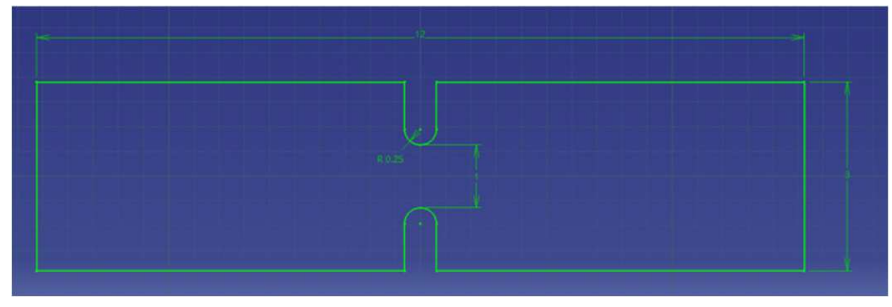

Fig. 5 Dimensions of a beam to be analyzed. The notch can be seen extending into the geometry of the beam, creating the desired d value, as well as maintaining the associated radius value. [3]

\section{C. $\quad$ Testing of Beams with W/d Ratios of 1.2 and 1.1}

For the last two experiments, with $W / d$ values of 1.2 and 1.1, an unforeseen problem occurred. While performing the first two experiments, the notch radii are constrained to be wholly within the dimensions of the beam. This is not possible for most of the trials conducted in the tests using $W / d$ values of 1.2 and 1.1, as the combined length of the notches and the imposed distance between them exceed the total width of the beam.

In order to determine the appropriate geometric configuration to keep the results consistent, numerous hypotheses are tested. Most do not yield results that are valid, as impossible geometric relationships are created. It is decided that the notch radii are taken from outside the beam's perimeter, as seen in
Fig. 6. This allows for the distance between the notches to remain consistent with the ratios the graph required. This is the only way to model the beams in order to conserve both necessary ratios.

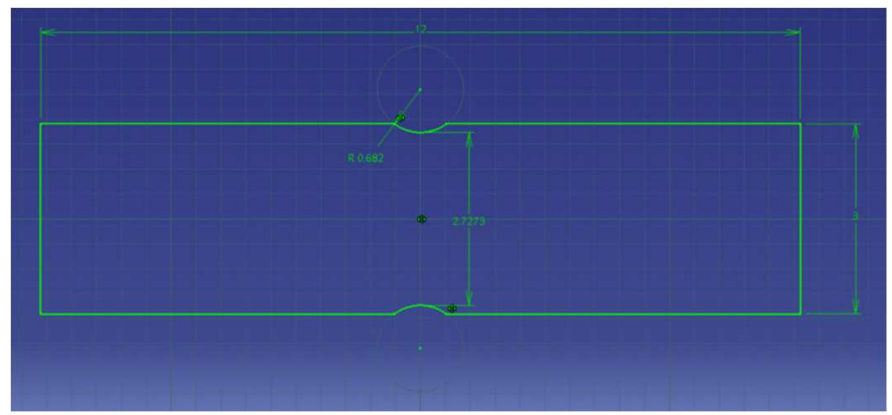

Fig. 6 Partial radius used to maintain the required $W / d$ and $r / d$ parameters. [3]

\section{D. $\quad$ Finite Element Analysis}

A mesh is applied to the beams to calculate the loading using Finite Element Analysis, FEA. When using a software program for FEA, a mesh convergence study must be performed to verify the results produced from the computation. The study consists of applying varying mesh sizes, starting with a comparatively large value, until the data generated begins to remain at constant value. While one could initially use a small mesh size without conducting the study, this method runs the risk of needing excess computation time for the extraneous precision in decimal places.

The mesh study completed shows that the convergence takes place at a mesh size of $2.54 \mathrm{~mm}(0.1 \mathrm{in})$, seen in Fig. 7. This determining factor is the last necessary before the analysis is run.

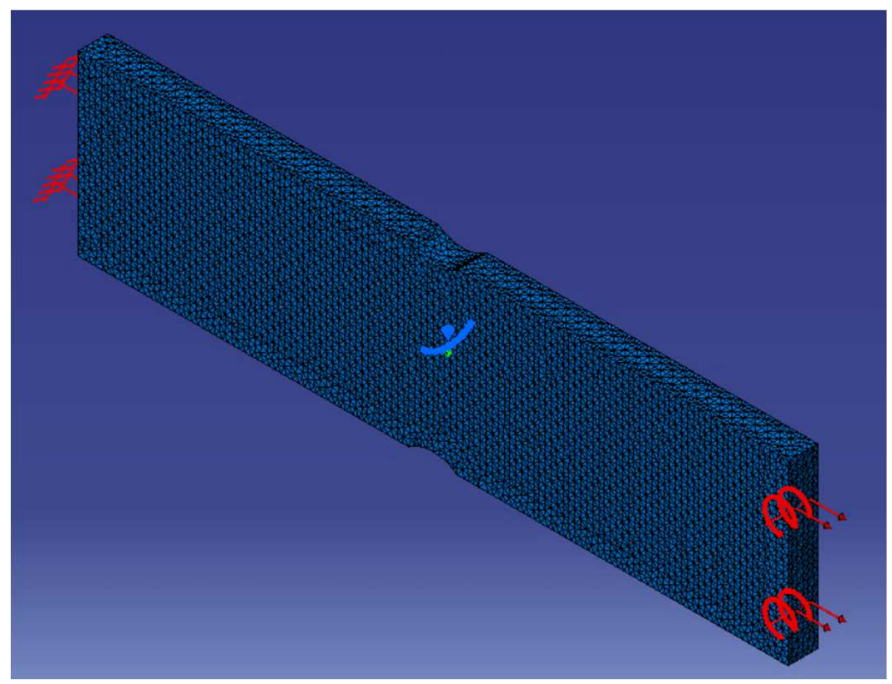

Fig 7 A mesh size of $2.54 \mathrm{~mm}$ (0.1 in) applied to the entire beam. [3]

$17^{\text {th }}$ LACCEI International Multi-Conference for Engineering, Education, and Technology: "Industry, Innovation, And Infrastructure for Sustainable Cities and Communities", 24-26 July 2019, Jamaica. 


\section{RESULTS}

A

Governing Equations Used to Calculate Stress

The calculation of average stress is performed using the minimum cross-sectional area of the part in question. Because of the combined loading, two equations must be utilized in order to find the value of average stress: the calculation of stress due to bending and the calculation of stress due to a surface load.

$$
\begin{gathered}
\sigma=\mathrm{Mc} / \mathrm{I} \\
\sigma=\mathrm{F} / \mathrm{A}_{\text {min }}
\end{gathered}
$$

Equation (1) is used to calculate the experienced stress from an applied moment. The moment, $M$, is multiplied by the minimum distance from the centroid of the beam to the notch radius, $c$. The value of $c$ can be found by dividing the distance between the notches in half, utilizing the value found at the minimum cross-sectional area. These values are then divided by the Moment of Inertia, $I$. The minimum cross-sectional area is used to find the value of $I$.

Equation (2) is used to calculate the experienced stress from an applied axial load. The force, $F$, is divided by the minimum cross-sectional area, $A_{\min }$. This value can be found by multiplying the thickness of the beam by the distance between the notches.

To find the total average stress a part experiences under combined loading, (1) and (2) are superimposed, which produces the following equation:

$$
\sigma_{\mathrm{AVG}}=\mathrm{Mc} / \mathrm{I}+\mathrm{F} / \mathrm{A}_{\min }
$$

Using (3), the average stresses are found for each of the four experiments. The values can be seen below, denoted by the prospective $W / d$ values.

$$
\begin{gathered}
\left(\sigma_{\mathrm{AVG}}\right)_{3}=165.47 \mathrm{MPa}(2,400 \mathrm{psi}) \\
\left(\sigma_{\mathrm{AVG}}\right)_{1.5}=5.171 \mathrm{MPa}(750 \mathrm{psi}) \\
\left(\sigma_{\mathrm{AVG}}\right)_{1.2}=3.64 \mathrm{MPa}(528 \mathrm{psi}) \\
\left(\sigma_{\mathrm{AVG}}\right)_{1.1}=3.185 \mathrm{MPa}(462 \mathrm{psi})
\end{gathered}
$$

\section{B. $\quad$ Calculating the Maximum Stress Using CATIA}

The values of maximum stress, shown in Table I, are found using the CATIA analysis data. Each beam's radius dimensions are modified five times, producing 20 different maximum stress values. The maximum stress is consistently found at the same location for each trial, the middle of the notch radius, as seen in Fig 8.

Equations (1), (2), and (3) are not the equations run by CATIA, however. The software uses an equation derived from the Von-Mises Criterion equation [1] which describes the stress using the Cauchy stresses, $\sigma_{\mathrm{x}}, \sigma_{\mathrm{y}}, \sigma_{\mathrm{z}}, \tau_{\mathrm{xy}}, \tau_{\mathrm{xz}}, \tau_{\mathrm{yz}}$.

$$
\sigma_{\mathrm{VM}}=\sqrt{\frac{1}{2}\left[\left(\sigma_{\mathrm{x}}-\sigma_{\mathrm{y}}\right)^{2}+\left(\sigma_{\mathrm{x}}-\sigma_{\mathrm{z}}\right)^{2}+\left(\sigma_{\mathrm{y}}-\sigma_{\mathrm{z}}\right)^{2}+6\left(\tau_{\mathrm{xy}}+\tau_{\mathrm{xz}}+\tau_{\mathrm{yz}}\right)\right]}
$$

The stress solved for is also called the effective stress, and the shear stress terms, $\tau_{\mathrm{xy}}, \tau_{\mathrm{xz}}, \tau_{\mathrm{yz}}$, are often dropped from the calculations when dealing solely with the principal stresses.

TABLE I

MAXIMUM STRESS VALUES (MPA)

\begin{tabular}{|l|l|l|l|l|}
\hline & $W / d=3$ & $W / d=1.5$ & $W / d=1.2$ & $W / d=1.1$ \\
\hline$r / d=0.05$ & 37.596 & 13.987 & 9.671 & 7.160 \\
\hline$r / d=0.10$ & 32.628 & 12.468 & 8.273 & 6.343 \\
\hline$r / d=0.15$ & 31.388 & 11.531 & 6.937 & 5.725 \\
\hline$r / d=0.20$ & 28.840 & 9.613 & 6.480 & 5.286 \\
\hline$r / d=0.25$ & 27.295 & 9.479 & 6.312 & 5.048 \\
\hline
\end{tabular}

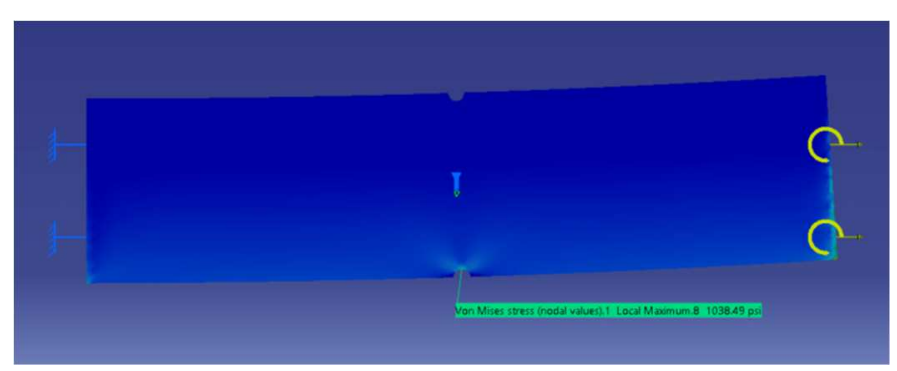

Fig 8 Location of maximum stress shown at the bottom notch. [3]

\section{Creation of the Stress Concentration Graph}

The Stress Concentration Graph, Fig. 9, is created using the calculated values of the Stress Concentration Factor, $K$. The values of $K$, shown in Table II, are calculated by dividing the experimental maximum stress values from each iteration by the analytical average stress values, (3). The $K$ values are plotted against the $r / d$ values to produce the four curves of the graph. Each curve represents a different $W / d$ value that is used.

TABLE II

STRESS CONCENTRATION FACTOR, K

\begin{tabular}{|l|l|l|l|l|}
\hline & $W / d=3$ & $W / d=1.5$ & $W / d=1.2$ & $W / d=1.1$ \\
\hline$r / d=0.05$ & 2.272008333 & 2.70488 & 2.656590909 & 2.247813853 \\
\hline$r / d=0.10$ & 1.971804167 & 2.411066667 & 2.272594697 & 1.991409091 \\
\hline$r / d=0.15$ & 1.89685 & 2.22984 & 1.905511364 & 1.797132035 \\
\hline$r / d=0.20$ & 1.742891667 & 1.859093333 & 1.780132576 & 1.659409091 \\
\hline$r / d=0.25$ & 1.6494875 & 1.83316 & 1.73375 & 1.584666667 \\
\hline
\end{tabular}



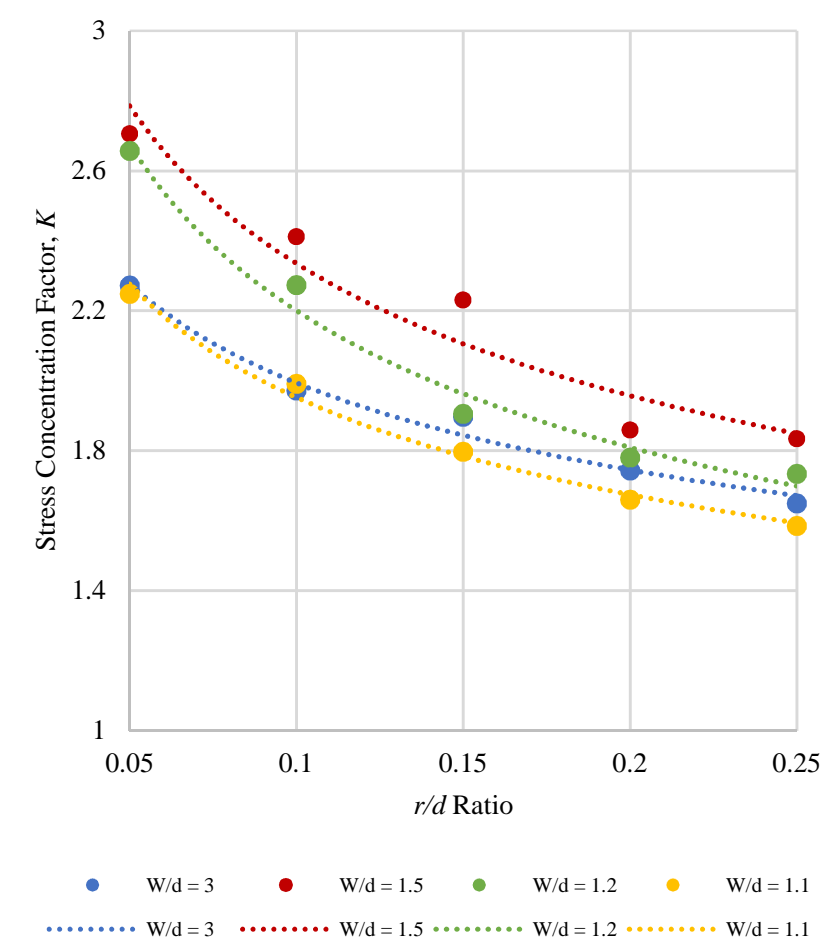

Fig. 9 Stress Concentration Graph: The plotted values of $K$ and their respective trend lines. Trend lines are used because of the inconsistencies of the values found.

\section{DISCUSSION}

As shown in the analysis, the stress concentration factor is dependent on the geometry of the structure. By applying a combined loading and studying its effects, the stress concentration factor is found for a beam with varying notch dimensions.

It is necessary to find both the average stress experienced by the beam and the maximum stress occurring at the location of the notch to achieve the results shown in Fig. 9. The ratio between the maximum and average stresses results in the value of the stress concentration factor. Each part is modeled and analyzed in CATIA to find its maximum stress values. Maximum stress is found occurring at the notch on the lower half of the beam. The reason the stress is maximum at the bottom notch is because of the orientation of the applied moment. The moment is applied in a way that causes the beam to bend upward, creating tension at this location, thereby causing a maximum stress at the bottom notch.

\section{A. Maximum Stress}

In order to find the value of maximum stress, an analysis tool is used to run the stress equation, (4), over a selected part of the plate. Using the overall stress analysis, the area of stress concentration is found and is used to find said peak. This feature studies the entire structure and labels the location of maximum stress.

However, the maximum stress produced through FEA is not found at the predicted location, rather, at the lower corner of the free end of the beam. This is due to the limited capabilities of the software being used. Because there are a finite number of elements being analyzed, sharp corners become difficult to compute. The mesh size would never be able to be small enough to account for the area in question. True $90^{\circ}$ corners do not exist, so in a physical experiment, no false values would be produced. Therefore, the sharp edge produced by the corner yields a false location of maximum stress [7]. This value is negated, and the true maximum stress is found at the bottom notch, which can be seen in Fig 10 below.

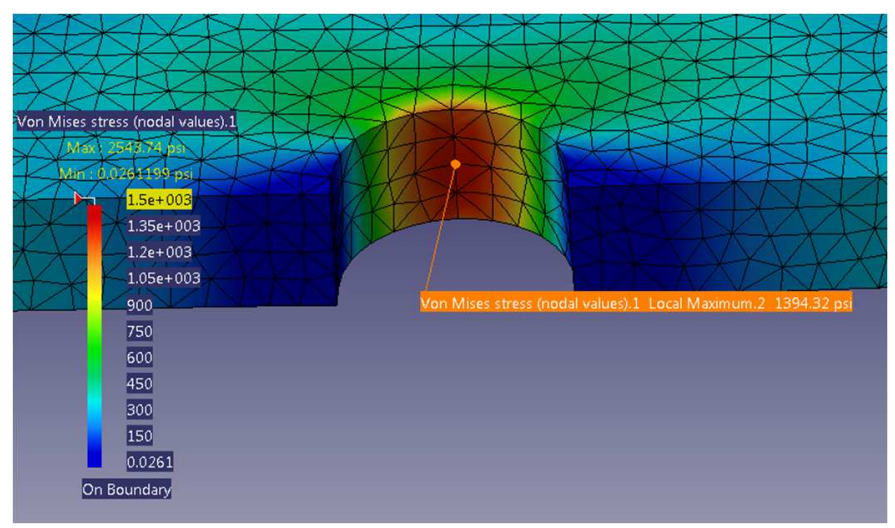

Fig. 10 Location of maximum stress of one iteration. [3]

\section{B. Average Stress}

Finding the average stress using the same simulation will be ideal since the results can be obtained using the same FEA method. However, using CATIA to find the average stresses for this experiment presents an issue because of the combined loading experienced by the beam. When analyzing loading conditions, CATIA solves for the stress occurring at each individual node throughout the structure. Because of this, the stress taken at any node uses the immediate cross-sectional area in reference to that node.

The analytical formula to calculate average stress (3), utilizes the value of the minimum cross-sectional area which, in this experiment, is the location of the notches. However, the maximum stress is experienced at this location, as stated previously. The location of average stress on the model analyzed using FEA is found by continuing across the horizontal axis relative to the location of the edge of the notch to a distance where the geometry does not affect the values, as seen in Fig 11.

Because of this inaccuracy, the average stress is found analytically, using (3). This formula uses the smallest crosssectional area, which is found in the values of the total area $A$, Moment of Inertia $I$, and distance to the centroid $c$.

17 ${ }^{\text {th }}$ LACCEI International Multi-Conference for Engineering, Education, and Technology: "Industry, Innovation, And Infrastructure for Sustainable Cities and Communities”, 24-26 July 2019, Jamaica. 


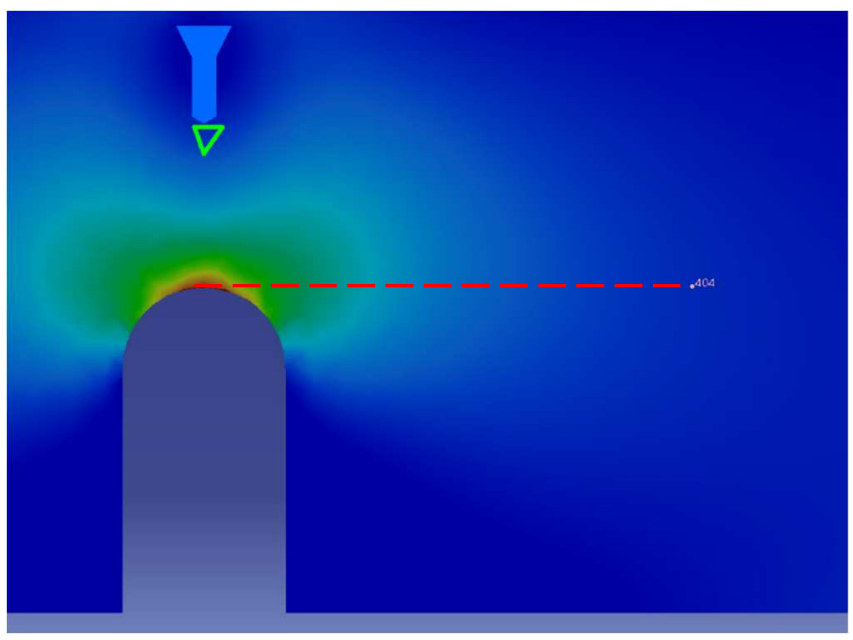

Fig. 11 Generation of the horizontal line following the height of the notch to the location of average stress found using CATIA

As seen in Fig. 11, the value CATIA calculates for the average stress of one of the iterations is $2.785 \mathrm{MPa}$ (404 psi). When solving for the average stress of the same iteration analytically, it is found to be $16.547 \mathrm{MPa}(2400 \mathrm{psi})$. The inaccuracy is due to CATIA not calculating the correct average stress. Because of the use of the immediate cross-sectional area, the values of $A, I$, and $c$ are changed to that of the area corresponding to that specific node, which in turn changes the produced value of stress. The value of $2.785 \mathrm{MPa}$ (404 psi) is the average stress a beam will experience if it does not have any discontinuities. One might think this issue may be resolved by looking at the stress in a point near the location of the smallest area. However, as stated previously, the notch geometries interfere with the results, producing incorrect values for stress. Ultimately, it is necessary to solve for the average stress analytically, and only the maximum stress is taken from the simulation results.

\section{C. $\quad$ Stress Concentration Factor}

The stress concentration factor relates the shape and size of the geometry of a beam with the stress produced by applied loads. As shown in Fig. 9, the stress concentration graph created from this experiment indicates how much the stress concentration factor increases when the ratio between the radii of the notches and the distance between the notches decreases. From this, we can deduce that the stress on a beam will increase as the notch size decreases. This is understandable as the smaller notch creates a more sudden discontinuity in the beam due to the imposed distance between them, as seen in the Fig. 12 and Fig. 13. While a larger notch creates a more substantial discontinuity, the smaller radius causes the stress to concentrate more abruptly, raising the maximum stress.

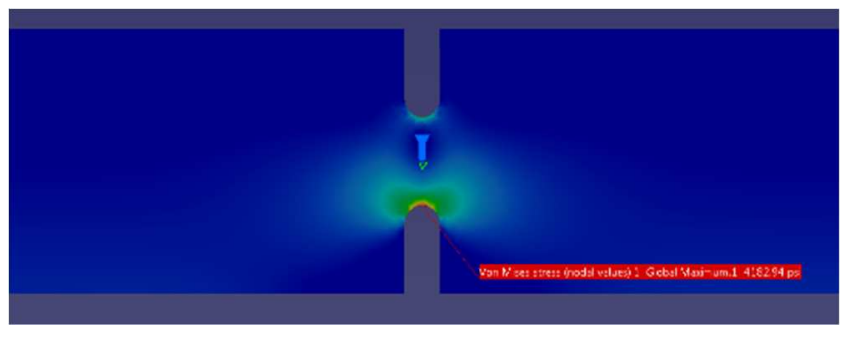

Fig. 12 A beam with a discontinuity of radius of 0.4 inches

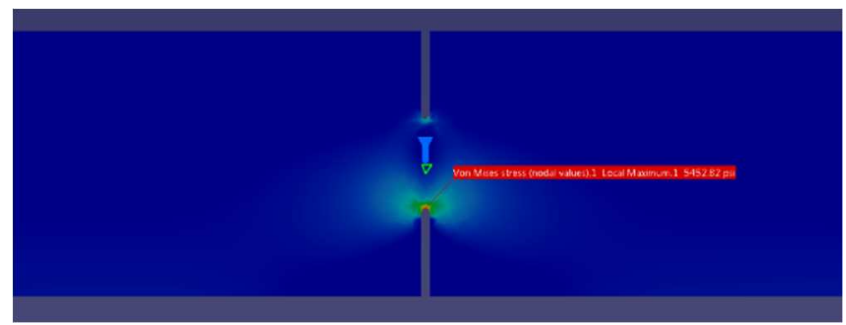

Fig. 13 Abeam with discontinuity of radius 0.1 inches

Fig. 9 shows the trend lines created from the $K$ values calculated from each experiment and iteration. As is expected, the curves produced from the tests using a higher $W / d$ ratio correspond to higher $K$ values. Unexpectedly, however, the highest $W / d$ ratio, 3 , yielded $K$ values that are comparable to the lowest $W / d$ ratio simulated. This is most likely due to the large discontinuity altering the way the stress is calculated, forcing the beam to act as a plate, bending and experiencing the load differently. A beam behaves as a one-dimensional element when experiencing loading, so (1) can be used to produce accurate results for the values of stress. When (1) is applied to find the stress of a plate, the results will incorporate error due to the formula. Therefore, it is assumed that the $K$ values and the curve created from the experiment using a $W / d$ ratio of 3 are outliers. The assumption is made as the values do not follow the trend created from the other data, as well as the trend established from the pre-existing and accepted stress concentration graphs used for axial or bending loading.

When comparing the results of the experimental graph, Fig. 9, to the two published graphs for axial loads and bending loads, Fig. 3 and Fig. 4, the results for the stress concentration factor for combined loading seem to be an average of the values from the two other graphs. The specimen with a $W / d$ ratio of 1.1 and an $r / d$ ratio of 0.15 have an estimated $K$ value of 1.7 on the bending moment graph and 1.9 on the axial loading graph. The average of these two values is 1.8 , the $K$ value produced from the experiments performed using both axial and bending loads. The same hypothesis is tested using the $W / d$ ratio of 1.5 and the $r / d$ ratio of 0.15 . The $\mathrm{K}$ values from the published graphs of axial and bending loads are 1.8 and 2.3, respectively. While the average of these to values is 2.05 , the value on the combined loading graph is seen to be about 2.1 , a

$17^{\text {th }}$ LACCEI International Multi-Conference for Engineering, Education, and Technology: "Industry, Innovation, And Infrastructure for Sustainable Cities and Communities", 24-26 July 2019, Jamaica. 
negligible difference of 0.05 which is likely due to the estimation involved when studying the chart.

As seen in Fig. 9, the trendline created from this ratio is inconsistent with the other curves. The curves from the ratios of $1.1,1.2$, and 1.5 have increasing $K$ values, respectively. The curve using the $W / d$ value of 3 produces $K$ values similar to the values from the experiments performed using both 1.1 and 1.2 as the $W / d$ ratio. This is likely due to the differences in the $W / d$ values used in the graph for axial loading and the graph for bending. The $W / d$ values on the stress concentration graph due to bending forces does not exceed 1.5. Beyond that value, all the $K$ factors remain consistent to one trendline; any chosen $W / d$ value has the same stress concentration factor in reference to their respective $r / d$ values. However, when conducting the experiments for the stress concentration factor due to combined loading, a $W / d$ ratio of 3 is used. This could have created the inconsistency in the relationships of the curves on the stress concentration graph using combined loading. It is possible that when a $W / d$ ratio greater than 3 is used, the values of $K$ due to the bending force component of the combined loading produce inconclusive results. Therefore, this is another reason the curve for the stress concentration factors using a $W / d$ ratio of 3 under combined loading is considered an outlier.

\section{CONCLUSION}

Having the necessary charts, graphs, and formulae make an engineer's job a little further from impossible. One such important tool is the stress concentration graph. However, the graph in question does fall short when combined loading is considered. CATIA is an excellent tool to utilize when studying the weakest points on a structure because of simulations the program can perform. These simulations include the ones performed in this experiment, which study high stress concentrations due to changes in the geometry. Although it is possible to find very accurate results of maximum stress using given stress concentration factor graphs, it is also worth noting that many structures do not have tables from where a stress concentration factor can be obtained, especially beams and rods that are designed for unique purposes. CATIA solves this issue by running simulations to determine these unknown variables. Using CATIA's application of finite element analysis, combined with a handful of formulae, a stress concentration graph is successfully generated and can be used by anyone so inclined to find the stress in a notched specimen undergoing a combined loading. After comparing this experimental chart to the published charts developed for only axial loading or bending moment, the trends of the experimental graph were consistent relative to those of the published charts. The exception was the curve using a $W / d$ value of 3 , which was considered an outlier.

\section{FUTURE WORK}

In the future, tests will be performed on physical specimens using a tensile testing machine. Because this machine cannot produce the effects of combined loading, the specimen will be designed in a manner that allows for the axial load to be transformed into a bending moment. By continuing this research using real specimen, the experimental values of the stress concentration factor can be confirmed or challenged. If challenged, further analyses will be run both through FEA and physical experiments to achieve a combined loading stress concentration graph that will be a great boon to any engineer.

\section{ACKNOWLEDGMENT}

The authors would like to thank Vaughn College of Aeronautics and Technology as well as the Engineering Department for their continued support in the furthering of the academic excellence of its student body. A thank you is also directed towards Dr. Hossein Rahemi, Department Chair of Engineering and Technology, and to Dr. Yougashwar Budhoo, the advisor to the authors of this paper.

\section{REFERENCES}

[1] "Analysis of a Bent Rod with Solid Elements." CATIA V5 FEA Tutorials, by Nader G. Zamani, Release 21 ed., Schroff Development Corp., 2012, pp. 2-16.

[2] “Appendix A.” Shigley's Mechanical Engineering Design, by Joseph Edward. Shigley and Charles R. Mischke, Ninth ed., McGraw-Hill, 2011, pp. 1026-1027.

[3] CATIA. Computer software. Vers. V5-6R2018. Vélizy-Villacoublay: Dassault Systemes, 2018.

[4] Beer, Ferdinand P., et al. Mechanics of Materials. Seventh ed., McGrawHill Education, 2015.

[5] Pilkey, Walter D. Peterson's Stress Concentration Factors. Second ed., John Wiley \& Sons, Inc. 1997.

[6] Nagpal, Shubhrata, et al. "Stress Concentration and Its Mitigation Techniques in Flat Plate with Singularities - A Critical Review." Engineering Journal, vol.16, no.1, 1 Jan. 2012, pp. 1-16., doi:10.4186/ej.2012.16.1.1.

[7] Gedeon, Mike. "Effects of Stress Concentration on Fatigue Life." Materion Brush Performance Alloys | Technical Tidbits, vol. 60, Dec. 2013.

$17^{\text {th }}$ LACCEI International Multi-Conference for Engineering, Education, and Technology: "Industry, Innovation, And Infrastructure for Sustainable Cities and Communities”, 24-26 July 2019, Jamaica. 